American Journal of Applied Sciences 6 (1): 43-47, 2009

ISSN 1546-9239

(C) 2009 Science Publications

\title{
Effect of Partial Orientation in [100] Direction on the Magnetic Properties of Co-Ferrite Prepared from Nano Particles
}

\author{
H.M. El-Sayed \\ Department of Basic Science, Faculty of Engineering, Misr University for Science and \\ Technology Cairo, Egypt
}

\begin{abstract}
Chemical co-precipitation method was used for the preparation of Co-ferrite nano particles. The particle size was about $18 \mathrm{~nm}$. A magnetic anisotropy of Co-ferrite could be increased by applying an external magnetic field during the pressing of the nano particles before the final sintering. This anisotropy enhanced the squareness and the coercivity of investigated samples.
\end{abstract}

Key words: Magnetic properties of Co-ferrite, nano particles, magnetic anisotropy

\section{INTRODUCTION}

Co-ferrites have attracted much attention in recent years as one of the candidates for high density magnetic recording and magneto-optical recording media because of their unique physical properties such as high Curie temperature, large magnetic anisotropy, moderate magnetization, excellent chemical stability and large Kerr and Faraday rotations ${ }^{[1,2]}$. However, the important problems, that the researchers are concerned with, are how to make Co-ferrite which has high coercivity, perpendicular anisotropy and small grain size for high density magnetic recording media.

Many ways are used for improving the magnetic properties of Co-ferrite. One of these methods is the divalent ions substitution e.g., $\mathrm{Ni}, \mathrm{Zn}$ and $\mathrm{Cd}$ on the account of Co ions ${ }^{[3,4,5]}$. But in fact this way gave a limited enhancement in the magnetic properties of Coferrite because the produced particles have large size and the formation of super-paramagnetic clusters.

One of the promising methods to improve the magnetic properties of Co-ferrite is the preparing of the material from its nano particles where, as the particle size decreases the magnetic coercivity increases ${ }^{[6]}$.

The objective of the present research is to improve the magnetic properties of Co-ferrite by making an induced magnetic anisotropy during the preparation of the samples from its nano particles. This will be done by applying an external magnetic field during pressing the powder of magnetic nano particles of Co-ferrite before the final sintering.

\section{PREPARATION OF THE SAMPLES AND EXPERIMENTAL TECHNIQUES}

Co-ferrite nano particles powder was prepared by Chemical co-precipitation method. A pure chemical reagents of $\mathrm{Fe}_{2}\left(\mathrm{SO}_{4}\right)_{3} .5 \mathrm{H}_{2} \mathrm{O}$ and $\mathrm{CoSO}_{4}$ were first dissolved in bi-distilled water and the $\mathrm{Co} / \mathrm{Fe}$ molar ratio was fixed to $1 / 2$. An alkaline solution $(\mathrm{NaOH})$ was added to the salt solution until the $\mathrm{pH}$ was adjusted to 12.5. The solution was heated at $90^{\circ} \mathrm{C}$ with continuous stirring for $1 \mathrm{~h}$. The co-precipitated powder was filtered and washed many times with bi-distillated water and dried in an oven in air atmosphere at $120^{\circ} \mathrm{C}$.

For preparing the bulk sample, the powder was pressed, in tablet (for x-ray and porosity measurements) and cubic ( for magnetization measurements) shapes, at $3 \times 10^{8}$ Pascal. The samples were pressed in the presence of external magnetic field $\left(\mathrm{H}_{\text {ext }}\right)$ which was parallel to the pressing direction. Four samples were prepared at different values of the external field $\left(\mathrm{H}_{\text {ext. }}=0.0\right.$, $12 \mathrm{KA} / \mathrm{m}, 16 \mathrm{KA} / \mathrm{m}$ and $20 \mathrm{KA} / \mathrm{m})$. The final sintering temperature was $1000^{\circ} \mathrm{C}$ for $6 \mathrm{~h}$.

$\mathrm{X}$-ray diffraction patterns were performed using a diffractometer of type X-Pert Graphics and identified with $\mathrm{Cu}-\mathrm{K}_{\alpha}$ radiation. The theoretical $\mathrm{x}$-ray density $\left(\mathrm{d}_{\mathrm{x}}\right)$ of the samples was calculated using the formula $\left(\mathrm{d}_{\mathrm{x}}=\right.$ $8 \mathrm{M} / \mathrm{Na}^{3}$ ) where, $\mathrm{M}$ is the molecular weight, $\mathrm{N}$ is Avogadro's number and a is the lattice parameter. The density (d) of each sample was measured in bi-distilled water using Archimedes principle. The porosity percentage $\mathrm{P}(\%)$ was calculated according to the relation

$$
\mathrm{P}=100\left(1-\mathrm{d} / \mathrm{d}_{\mathrm{x}}\right) \% .
$$

The magnetization (M), at room temperature and the hysteresis parameters were measured using the vibrating sample technique. The magnetizing field ranged from 0.0 up to $12 \mathrm{~K}$ Oe.

The particle size $\left(D_{\mathrm{hkl}}\right)$ of the powder sample was measured from the x-ray chart according to DebyeSherrer formula ${ }^{[7]}$ which is given by, 
Am. J. Applied Sci., 6 (1): 43-47, 2009

$$
\mathrm{D}_{\mathrm{hkl}}=0.9 \lambda / \beta \cos \theta
$$

where, $\lambda$ is the wave length of the used $\mathrm{x}$-ray $(\lambda=0.154$ $\mathrm{nm}), \beta$ is the half width and $\theta$ is the half diffraction angle. The particle size was determined by taking the average of the strongest peaks $\mathrm{D}_{220}, \mathrm{D}_{311}, \mathrm{D}_{400}, \mathrm{D}_{511}$ and $\mathrm{D}_{440}$. Also, the Scanning electron microscope (SEM) was used for measuring the grain size of the investigated samples.

\section{RESULTS AND DISCUSSION}

X-ray analysis: X-ray diffraction of the powder sample is shown in Fig. 1a. It is obvious that, the prepared sample from the chemical co-precipitation method has one cubic spinel phase. The lattice parameter was found to be about $8.383 \mathrm{~A}^{\circ}$ which is in good agreement with that reported by Armulmurugan et al. and Pandya et al. ${ }^{[8,9]}$. Also, from $\mathrm{x}$-ray pattern, the average particle size of the powder sample is found to be about $18 \mathrm{~nm}$. This result confirms that, the prepared sample has nano particle size. Figure 1b shows the image of SEM of the powder sample. It is noticed that, the powder sample has nano size particles in the range of $12 \mathrm{~nm}$ which is in a good agreement with $\mathrm{x}$-ray measurements.

The x-ray diffraction patters of the bulk samples are shown in Fig. 2. It is clear that, all patterns have single spinel phase. It was found that, the lattice parameter of all samples is in the same order of the powder sample. Furthermore, it is valuable to note that, the relative intensities of (100) and (400) planes increase with increasing the applied magnetic field during pressing the samples $\left(\mathrm{H}_{\text {ext }}\right)$ while, at the same time, the relative intensities of the (220) and (440) decrease. This may be discussed as follows:

As the average particle size of the powder sample is much less than the magnetic domain size, one may conclude that, each particle has one domain i.e., each particle could be considered as a permanent magnet. Moreover, It is well known that, the easy magnetization axis of Co-ferrite is [100] where, this direction has the highest magnetic anisotropy ${ }^{[10,11]}$. Thus the domain direction of each particle prefers to be in [100] direction. Thus, by applying the external field, the nano particles will rotate in the direction of the applied field i.e., the crystallographic direction of the powder will have partial orientation in [100] direction, Fig. 3. By increasing the applied field $\left(\mathrm{H}_{\mathrm{ext}}\right)$, the degree of orientation in [100] direction will increase. This orientation causes the increase of the relative intensity of (100) plane. As the plane (400) is parallel to (100) then, the relative intensity of this plane also increases on the account of the two parallel planes (220) and (440).



Fig. 1: (a) X-ray diffraction pattern and (b) SEM of Co-Ferrite nano-particles.

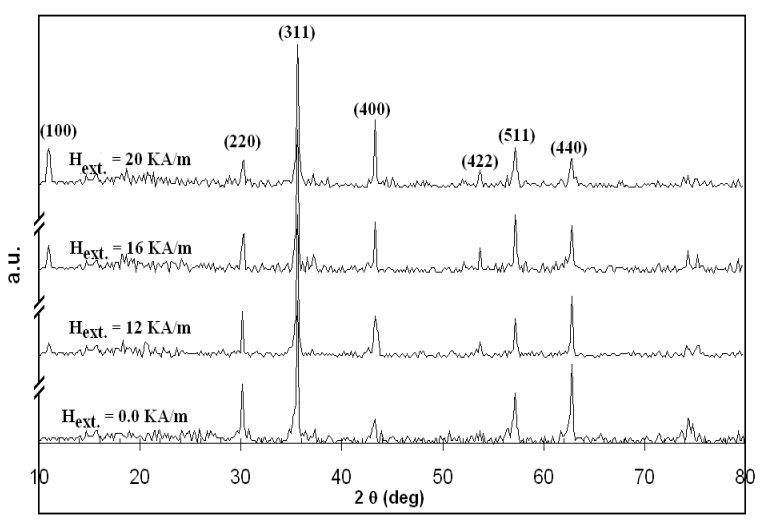

Fig. 2: X-ray Diffraction Patterns of Co-Ferrite subjected to external applied magnetic field $\left(\mathrm{H}_{\mathrm{ext}}.\right)$.

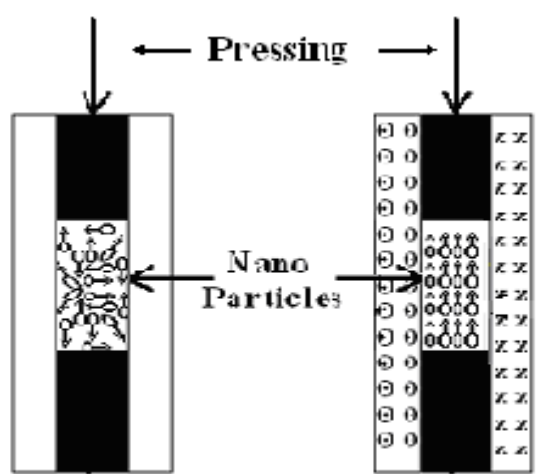

\section{Magnetic field(OFF) Magnetic Field (ON)}

Fig. 3: An illustration figure shows the rotation of the magnetic nano-particles due to the application of magnetic field during pressing the samples. 

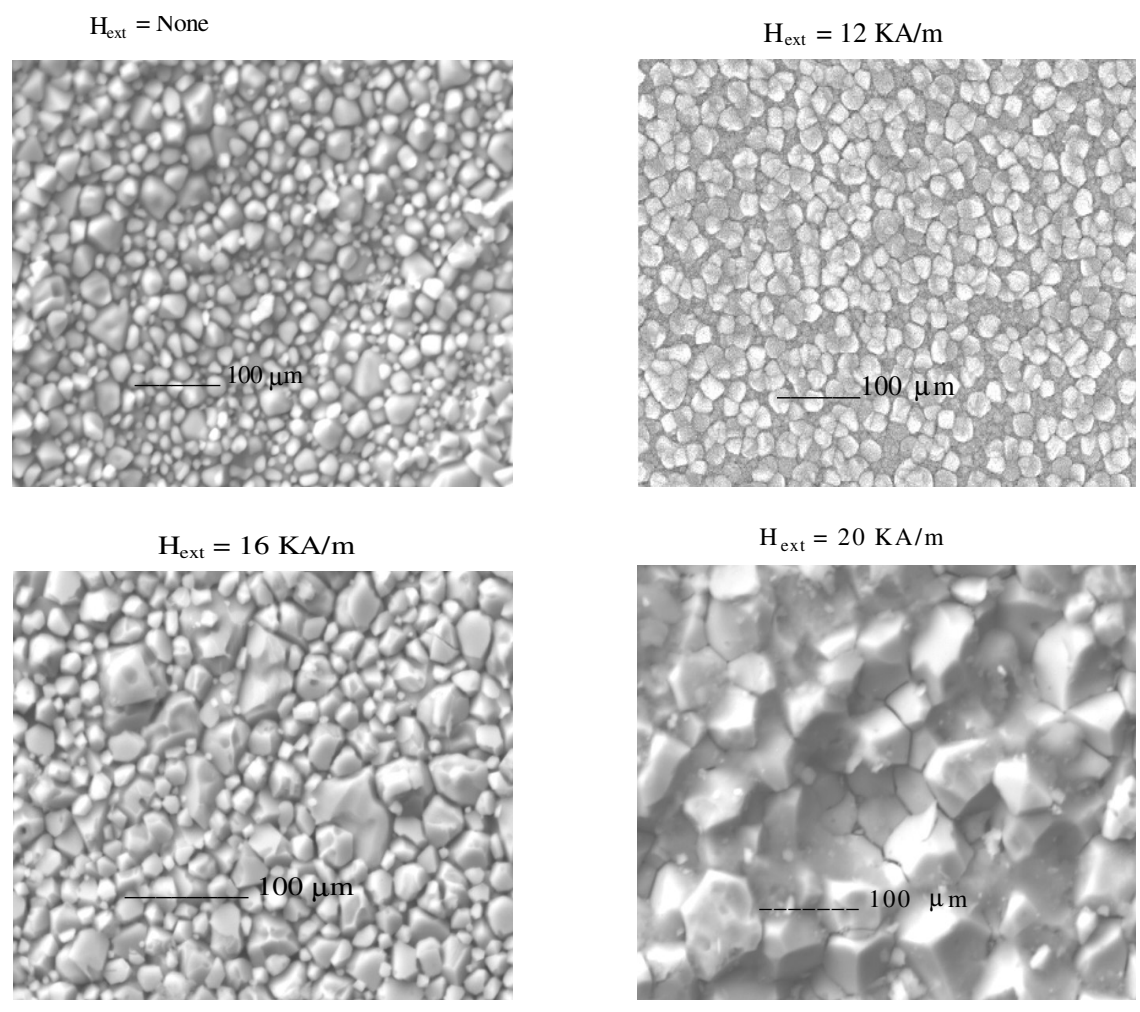

Fig. 4: SEM images for Co-ferrite at different $\mathrm{H}_{\text {ext. }}$

Figure 4 shows the SEM images of the investigated samples. The average grain sizes of the samples at different $\mathrm{H}_{\mathrm{ext}}$ are shown in Table 1. It is noticed that, the average grain size increases with increasing $\mathrm{H}_{\text {ext. }}$. This increase of the grain size with the magnetic field $\left(\mathrm{H}_{\mathrm{ext} t}\right)$ could be attributed to the orientation effects of the nano particles with the applied magnetic field $\left(\mathrm{H}_{\text {ext. }}\right)$. From Table(1), it is also observed that, the porosity has an inverse relation with the applied field $\left(\mathrm{H}_{\mathrm{ext} .}.\right)$ which may be attributed to the increase of the grain size.

Magnetization measurements: In fact, the enhancement of the degree of orientation of the Coferrite samples with the applied field $\left(\mathrm{H}_{\text {ext. }}\right)$ in [100] direction is expected to affect the magnetic properties of the investigated samples. Figure 5 shows the magnetization curve in the perpendicular and parallel directions to the surface of the sample at $\mathrm{H}_{\text {ext. }}=$ $20 \mathrm{KA} / \mathrm{m}$. It is clear that, the perpendicular direction is easy to be magnetized than the parallel one. This means that, there is magnetic anisotropy in the sample.

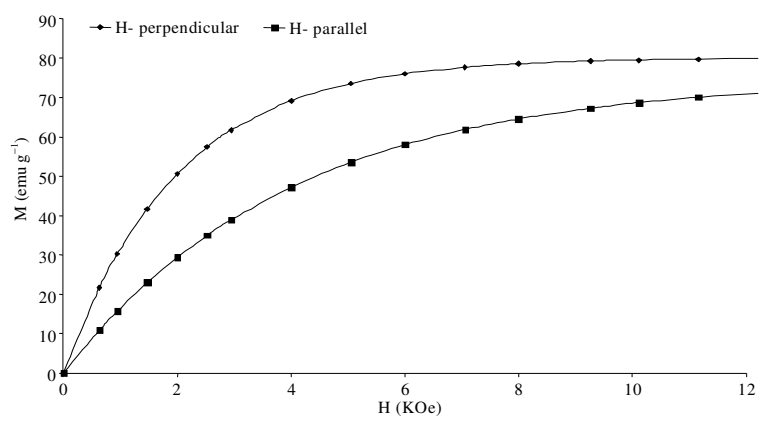

Fig. 5: Magnetization curve for the sample with $\mathrm{H}_{\text {ext. }}=20 \mathrm{KA} / \mathrm{m}$ in Parallel and perpendicular directions to the surface of the sample

This is attributed to the degree of orientation of the samples in [100] direction. The magnetization curves of the four samples in perpendicular direction to the surface of the sample are shown in Fig. 6. It is obvious that, the sample which is pressed under higher magnetic field is easier to be magnetized i.e. the anisotropy becomes higher. 
Am. J. Applied Sci., 6 (1): 43-47, 2009

Hysteresis parameters: The hysteresis loops of the four investigated samples are shown in Fig. 7. The

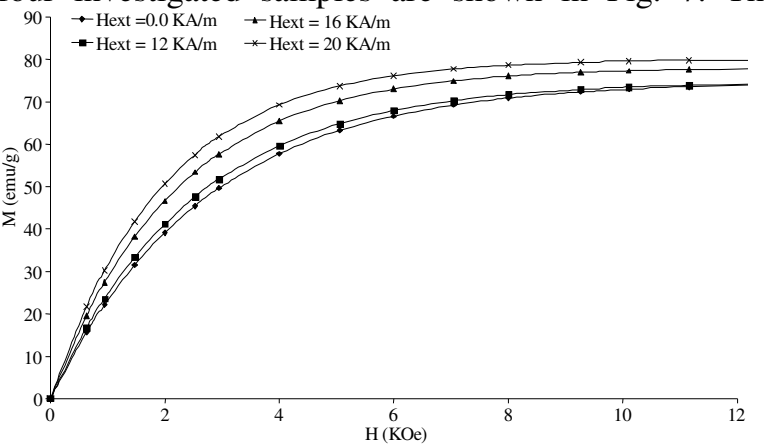

Fig. 6: Magnetization Curves for all samples

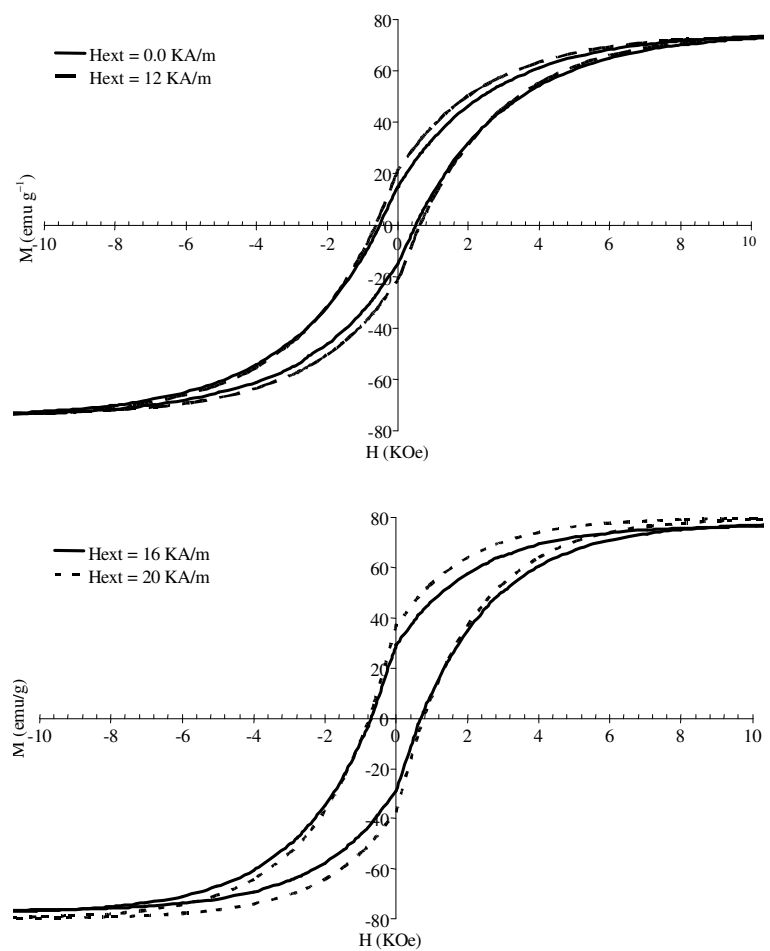

Fig. 7: Hysteresis loops of Co-ferrite samples at different $\mathrm{H}_{\mathrm{ext}}$

hysteresis parameters, the saturation magnetization $\left(M_{s}\right)$, remanance $\left(M_{r}\right)$, ratio $\left(M_{r} / M_{s}\right)$, which represents the squareness, and the coercivity $\left(\mathrm{H}_{\mathrm{c}}\right)$ for the all samples are shown in Table 1. It is obvious that, the ratio $\left(\mathrm{M}_{\mathrm{r}} / \mathrm{M}_{\mathrm{S}}\right)$ increases with increasing the applied field during the pressing. This means that there is an enhancement of the squareness of the samples. On the other hand, there is an increase of the coercivity with increasing $\mathrm{H}_{\mathrm{ext}}$. Since according to Brown`s relation ${ }^{[12]}$
Table 1: Grain size, porosity and the hysteresis parameters of CoFerrite

\begin{tabular}{lllllll}
\hline $\begin{array}{l}\text { Applied } \\
\text { Field }(\mathrm{KA} / \mathrm{m})\end{array}$ & $\begin{array}{l}\text { Grain Size Porosity } \\
(\mu \mathrm{m})\end{array}$ & $\begin{array}{l}\mathrm{M}_{\mathrm{s}} \\
(\%)\end{array}$ & $\begin{array}{l}\mathrm{M}_{\mathrm{r}} \\
(\mathrm{emu} / \mathrm{g})\end{array}$ & $\begin{array}{l}\left.\mathrm{M}_{\mathrm{r}} / \mathrm{M}_{\mathrm{s}} / \mathrm{g}\right) \\
(\%)\end{array}$ & $\begin{array}{l}\mathrm{Hc} \\
(\mathrm{Oe})\end{array}$ \\
\hline 0.0 & 11 & 12 & 74 & 19 & 25 & 330 \\
12 & 14 & 12 & 74 & 22 & 30 & 420 \\
16 & 20 & 10 & 77 & 28 & 36 & 630 \\
20 & 33 & 8 & 79 & 37 & 46 & 700 \\
\hline
\end{tabular}

$$
\mathrm{H}_{\mathrm{c}} \geq\left(2 \mathrm{~K}_{1} / \mu_{\mathrm{o}} \mathrm{M}_{\mathrm{s}}\right)
$$

where, $\mathrm{K}_{1}$ is the anisotropy constant. One can see that, the coercivity has a direct relation with the anisotropy constants $\mathrm{K}_{1}$. Therefore, the increase of $\mathrm{H}_{\mathrm{C}}$ with the applied field, during the pressing of samples, proves the increase of the magnetic anisotropy. This means that, the application of a magnetic field during the pressing of Co-ferrite nano particles before sintering could enhance the magnetic anisotropy without the need of substituting any other ions.

\section{CONCLUSIONS}

Co-ferrite nano particles of size $18 \mathrm{~nm}$ could be obtained by chemical co-precipitation method at low temperature. The application of an external magnetic field on the sample during pressing causes great effect on the magnetic properties of Co-ferrite. During this work an enhancement of the squareness of Co-ferrite was obtained. Also, the increase of the magnetic anisotropy due to the application of the magnetic field during the pressing causes an increase in the coercivity of the Co-ferrite.

\section{REFERENCES}

1. Fontijn, W.F.J., P.J. van der Zaag, L.F. Feiner, R. Metselaar and M.A. Devillers, 1999. A consistent interpretation of the magneto-optical spectra of spinel type ferrites. J. Appl. Phys., 85: 5100.

2. Suzuki, Y., 2001. Epitaxial spinel ferrite thin films. Ann. Rev. Mater. Res., 31: 265.

3. Rangel, A.M., T. Ogasawara and M.C. Nobrega, 2006. Investigation of sintered cobalt-zinc ferrite synthesized by co-precipitation at different temperatures: A relation between microstructure and hysteresis. Mat. Res., 9: 3.

4. Guillot, M., J. Ostorero, A. Marchand and A. Baret, 1988. Magnetic properties of Cadmiumcobalt ferrite. J. Phys. Colloques, 8: 923.

5. Niu, Z.P., Y. Wang and F.S. Li, 2006. Magnetic properties of nanocrystalline co-ni ferite. J. Mater. Sci., 41: 17. 
6. Yin, J.H., B.H. Liu, J. Ding and Y.C. Wang, 2006. High coercivity in nanostructured Co-ferrite thin films Bull. Mater. Sci., 29 (6): 573-580. () Indian Academy of Sciences 573.

7. Cuozhong Cao, 2004. Nano Structures and Nano Materials. Published by Imperial Collage Press, pp: 331.

8. Armulmurugan, R., B. Jeyadevan, G. Vaidyanathan and S. Sendhilnathan, 2005. Effect of zinc substitution on $\mathrm{Zn}$ ferrite nano particles prepared by co-precipitation. J. Mag. Mag. Mater., 288: 470.

9. Pandya, P.B., H.H. Joshi and R.G. Kulkarni, 1991. Bulk magnetic properties of $\mathrm{Co}-\mathrm{Zn}$ ferrites prepared by the co-precipitation method. J. Mater. Sci., 26 (20): 5509.
10. Jyh-Shinn Yang and Ching-Ray Chang, 1994. Magnetization curling in elongated heterostructure particles, Phys. Rev., B 49: 11877-11885.

11. Tsuchiya, T., H. Yamashiro, T. Sei ${ }^{1}$ and T. Inamura, 1992. Preparation of Spinel-Type Ferrite thin Films by the Dip-Coating Process and their Magnetic Properties. Yu, L.Q., L.J. Zheng and J.X. Yang, (Eds.), 27 (13): 2000.

12. Coey, J.M.D., 1996. Rare Earth Permanent Magnetism. Published by John Wiley and Sons, New York, pp: 220. 